\title{
Multiple Midfrontal Thetas Revealed by Source Separation of Simultaneous MEG and EEG
}

\author{
Marrit B. Zuure, ${ }^{1}$ Leighton B. Hinkley, ${ }^{2}$ Paul H.E. Tiesinga, ${ }^{1}$ Srikantan S. Nagarajan, ${ }^{2}$ and ${ }^{\circledR}$ Michael X Cohen ${ }^{1,3}$ \\ ${ }^{1}$ Donders Centre for Neuroscience, Radboud University, 6525 AJ Nijmegen, The Netherlands, ${ }^{2}$ Radiology Department, University of California at \\ San Francisco, San Francisco, California 94143, and ${ }^{3}$ Donders Centre for Medical Neuroscience, Radboud University Medical Center, 6525 AJ \\ Nijmegen, The Netherlands
}

Theta-band $(\sim 6 \mathrm{~Hz})$ rhythmic activity within and over the medial PFC ("midfrontal theta") has been identified as a distinctive signature of "response conflict," the competition between multiple actions when only one action is goal-relevant. Midfrontal theta is traditionally conceptualized and analyzed under the assumption that it is anitary signature of conflict that can be uniquely identified at one electrode (typically FCz). Here we recorded simultaneous MEG and EEG (total of 328 sensors) in 9 human subjects ( 7 female) and applied a feature-guided multivariate source-separation decomposition to determine whether conflict-related midfrontal theta is a unitary or multidimensional feature of the data. For each subject, a generalized eigendecomposition yielded spatial filters (components) that maximized the ratio between theta and broadband activity. Components were retained based on significance thresholding and midfrontal EEG topography. All of the subjects individually exhibited multiple (mean 5.89, SD 2.47) midfrontal components that contributed to sensor-level midfrontal theta power during the task. Component signals were temporally uncorrelated and asynchronous, suggesting that each midfrontal theta component was unique. Our findings call into question the dominant notion that midfrontal theta represents a unitary process. Instead, we suggest that midfrontal theta spans a multidimensional space, indicating multiple origins, but can manifest as a single feature at the sensor level because of signal mixing.

Key words: conflict processing; midfrontal cortex; multivariate analysis; response conflict; source separation; theta

Significance Statement

"Midfrontal theta" is a rhythmic electrophysiological signature of the competition between multiple response options. Midfrontal theta is traditionally considered to reflect a single process. However, this assumption could be erroneous because of "mixing" (multiple sources contributing to the activity recorded at a single electrode). We investigated the dimensionality of midfrontal theta by applying advanced multivariate analysis methods to a multimodal MEG/EEG dataset. We identified multiple topographically overlapping neural sources that drove response conflict-related midfrontal theta. Midfrontal theta thus reflects multiple uncorrelated signals that manifest with similar EEG scalp projections. In addition to contributing to the cognitive control literature, we demonstrate both the feasibility and the necessity of signal demixing to understand the narrowband neural dynamics underlying cognitive processes.

\section{Introduction}

Oscillatory activity is driven by rhythmically active neural populations (Buzsáki and Draguhn, 2004; Wang, 2010). The temporal properties of these oscillations can inform us about the functional properties of the generating neural populations, which

\footnotetext{
Received Feb. 7, 2020; revised July 22, 2020; accepted July 31, 2020.

Author contributions: M.B.Z., L.B.H., S.S.N., and M.X.C. designed research; M.B.Z. and M.X.C. analyzed data; M.B.Z. wrote the first draft of the paper; M.B.Z., L.B.H., P.H.E.T., S.S.N., and M.X.C. edited the paper; M.B.Z. and M.X.C. wrote the paper; L.B.H. and M.X.C. performed research; P.H.E.T. contributed unpublished reagents/ analytic tools.

M.B.Z. and M.X.C. were supported by European Research Council (ERC)-StG 638589

The authors declare no competing financial interests.

Correspondence should be addressed to Michael X Cohen at mikexcohen@gmail.com.

https://doi.org/10.1523/JNEUROSCI.0321-20.2020

Copyright $\odot 2020$ the authors
}

presumably implement the cognitive processes co-occurring with the oscillation (Womelsdorf et al., 2014). In this way, oscillatory markers of cognition can be used to understand cognition itself.

Midfrontal theta, characterized as brief theta-frequency (5$7 \mathrm{~Hz}$ ) power increases at midfrontal EEG electrodes in humans, is well established as a marker of response conflict: competition between concurrently activated responses of which only one is goal-relevant (Botvinick et al., 2001). The power of the theta burst covaries with reaction time and is thought to index the level of subjective conflict (Cohen and Cavanagh, 2011). Response conflict theta is spectrally dissociable from errorrelated and other types of midfrontal theta (Cohen, 2014a; see, e.g., Kahana et al., 2001; van de Vijver et al., 2011; Cohen and van Gaal, 2014). It is non-phase-locked to stimulus or response 
(Cohen and Donner, 2013), suggesting that conflict enhances ongoing oscillations in the medial frontal cortex (MFC), where the signal is estimated to originate (Ridderinkhof et al., 2004). Task-relevant regions phase-lock to conflict theta (Hanslmayr et al., 2008; Cohen and Cavanagh, 2011), indicating a driving or coordinating role for conflict theta in recruiting brain networks for conflict processing. Despite extensive characterization of the signal itself, its generating neural mechanisms remain unidentified, with existing neurobiological accounts (Cohen, 2014a) being speculative at best.

Lacking mechanistic explanations, response conflict theta is assumed to be a unitary phenomenon: that is, it consists of a single signal that varies over time and correlates with the amount of conflict. Indeed, EEG and fMRI studies of response conflict theta typically capture a single source (Ridderinkhof et al., 2004), and much of the contemporary literature reflects the unitary assumption (Botvinick et al., 2001; Nigbur et al., 2011; Pastötter et al., 2013; Cavanagh and Frank, 2014; Verguts, 2017), although Töllner et al. (2017) reported two theta sources in PFC. However, this assumption may be erroneous. Because electrical signals mix linearly and instantaneously (Nunez and Srinivasan, 2006), response conflict theta may consist of multiple independent signals but appear as a singular phenomenon at the sensor level. Similarly, the "single blob" observations typical in individual fMRI studies may hide multiple conflict-related sources, obscured by spatial normalization, smoothing, and cross-subject averaging.

In this study, we questioned the assumption that response conflict theta reflects a unidimensional neural process. We applied a feature-driven multivariate source separation method that is optimized for determining whether narrowband activity reflects the linear summation of independent sources or a single (nonlinearly separable) source (de Cheveigné and Parra, 2014). This method (generalized eigendecomposition [GED]) outperforms linear decomposition methods, such as principal component analysis (PCA) and independent component analysis (ICA), at source separation (Nikulin et al., 2011; Cohen, 2017a; Cohen and Gulbinaite, 2017), in part because it can selectively target data features of interest.

We applied theta-targeted source separation to simultaneous MEG and EEG recorded during the "Simon task," a task commonly used to induce response conflict (Leuthold, 2011). Our multimodal MEG/EEG dataset lends itself especially well to source separation, in part because of the high sensor count and in part because MEG and EEG are sensitive to nonredundant (i.e., predominantly tangentially vs radially oriented) neural sources. All 9 subjects exhibited multiple linearly separable sources that contributed to sensor-level midfrontal theta. Inferential statistics confirmed that these sources explained significant amounts of variance in the data, and further analysis revealed that sources were temporally uncorrelated and asynchronous, implying uniqueness. Midfrontal theta thus does not appear to be a singular phenomenon, but rather a linear combination of many theta-frequency signals with overlapping topographies.

We conclude that midfrontal theta spans a multidimensional space, indicating multiple origins, and that it can manifest as a single feature at the sensor level because of signal mixing. We speculate that these dimensions reflect independent computations during conflict processing.

\section{Materials and Methods}

Subjects. Ten subjects (7 female) from the University of California San Francisco (UCSF) community participated in this study. The
UCSF Internal Review Board approved the study, and all research was performed in accordance with UCSF Internal Review Board regulations. All subjects gave written informed consent. Inclusion criteria were the absence of psychiatric and neurologic disorders, absence of substance dependence or substance abuse, and MRI safety criteria.

Stimulus, task, and study design. Subjects performed a conflictinducing Simon task (see Fig. 1A), previously described by Cohen and Ridderinkhof (2013). A colored circle was presented on the monitor, to the left or right of the fixation cross. Subjects were instructed to perform a left or right button press in response to the stimulus color while ignoring the stimulus location. Response conflict was present on trials where the target response was contralateral to the stimulus location ("incongruent trials"). Conversely, no response conflict was present on trials where the target response was ipsilateral to the stimulus location ("congruent trials"). Subjects completed 1500 trials in blocks of 60 .

$M E G / E E G$ recording and preprocessing. MEG data were recorded in a shielded room using a whole-head 275 axial gradiometer MEG system with third-order gradient correction (CTF MEG International Services) at a sampling rate of $1200 \mathrm{~Hz}$. Three MEG sensors (MLF62, MLF64, MLT16) were inactive; 56 EEG electrodes were placed on the scalp according to the 10-20 system. MEG/EEG data were acquired under a bandpass filter of $0.001-300 \mathrm{~Hz}$. Third-order gradient noise correction filters were applied to the MEG data and corrected for a direct-current offset, using CTF-provided routines.

Offline MEG/EEG cleaning and processing were performed with custom MATLAB scripts (MATLAB 2014a, The MathWorks) and the EEGlab toolbox (Delorme and Makeig, 2004). Data were segmented into epochs running from $1.5 \mathrm{~s}$ before to $2.5 \mathrm{~s}$ after stimulus onset. Trials were visually inspected, and excessively noisy trials were rejected. Trials were classified into "congruent" and "incongruent" task conditions. As the recent history of conflict modulates reaction times (Gratton et al., 1992) and electrophysiological effects (Pastötter et al., 2013), potentially reflecting divergent cognitive processes, analyses were performed only on trials following a congruent trial (compare Cohen and Ridderinkhof, 2013). Error trials were excluded from analysis. This left $534 \pm 63.7$ trials per subject, with a minimum of 425. MEG/EEG data were further cleaned using ICA in EEGlab. Components with clearly identifiable nonbrain artifacts, such as eye blinks or heartbeats, were removed.

Sensor-level time-frequency analysis. Data recorded at midfrontal EEG electrode FCz were time-frequency decomposed through trial $\times$ trial convolution with 40 complex Morlet wavelets. Morlet wavelets were constructed as follows (see also Cohen, 2014b):

$$
\begin{gathered}
\Psi_{f}=e^{i 2 \pi f t} e^{-t^{2} / 2 s^{2}} \\
s=\frac{n}{2 \pi f}
\end{gathered}
$$

where $t$ is time, $f$ is frequency (logarithmically spaced from $2-20 \mathrm{~Hz}$ ), $s$ is the SD of the Gaussian that modulates the complex sine wave, and $n$ is the number of wavelet cycles (logarithmically spaced from 4 to 10). The number of cycles per wavelet governs the trade-off between temporal and spectral precision.

Frequency-specific power was extracted as the squared amplitude of the absolute analytic signal resulting from the convolution. Power was then $\mathrm{dB}$-scaled relative to a trial-averaged -500 to $-100 \mathrm{~ms}$ baseline, $0 \mathrm{~ms}$ being stimulus onset. One of 10 subjects exhibited uncharacteristically weak theta-frequency power at electrode $\mathrm{FCz}$ and was excluded from further analysis, leaving 9 subjects ( 7 female).

Multivariate-guided source separation. The source separation method used (GED), also detailed by de Cheveigné and Arzounian (2015) and Cohen (2017b), allows for the selection of features of interest in the data. Two channel $\times$ channel covariance matrices $\mathbf{S}$ and $\mathbf{R}$ were constructed, based on the channels $\times$ time signal of interest $\mathbf{X}_{\mathrm{s}}$ and the reference signal $\mathbf{X}_{\mathrm{r}}$ as follows: 
A

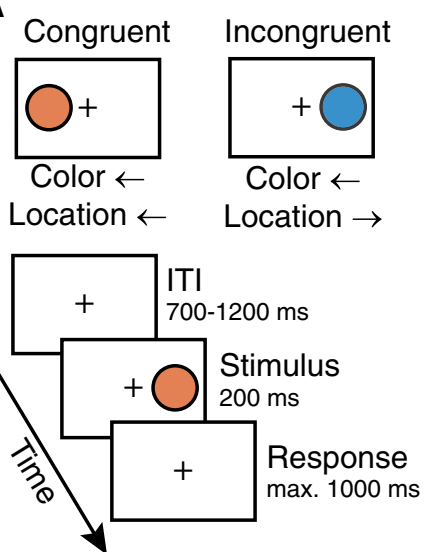

C

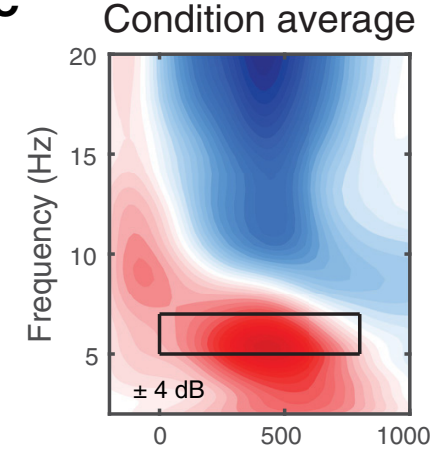

Time (ms)

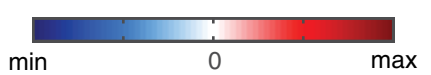

D

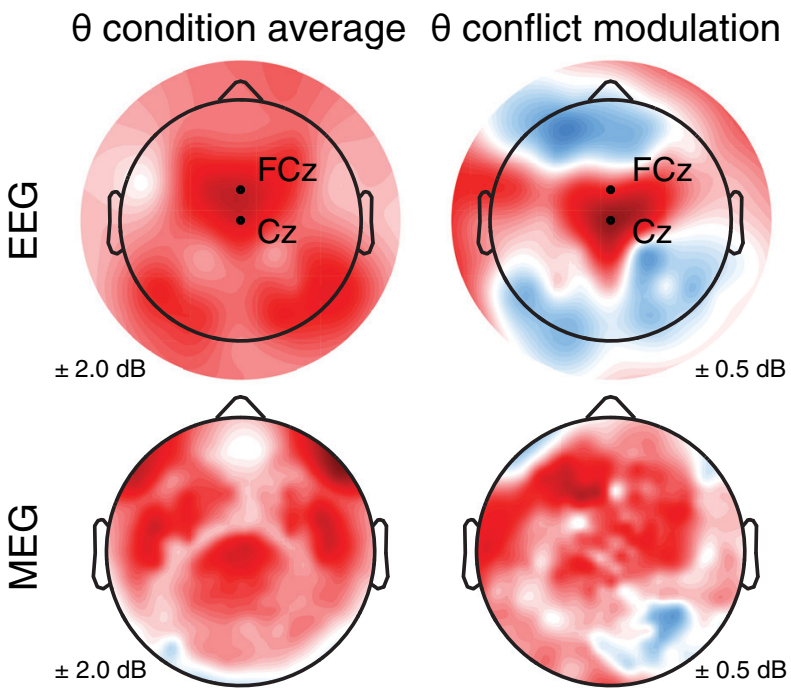

Figure 1. Task design, behavioral effects, and sensor-level effects. $\boldsymbol{A}$, Subjects performed a Simon task with congruent (one direction cued) and incongruent, that is, conflict-inducing (two directions cued) trials. $\boldsymbol{B}$, Reaction times ( \pm SEM) per trial type show the expected Simon effect (faster responses on congruent than incongruent trials) and expected modulation of conflict effects by previous conflict. $\mathrm{CC}$, Congruent following congruent; $\mathrm{Cl}$, incongruent following congruent; iC, congruent following incongruent; il, incongruent following incongruent. $\boldsymbol{C}$, Condition-average and conflict modulation of time-frequency power at electrode FC. Black rectangle represents the time-frequency window used to create the covariance matrices for the GED. D, Subject-averaged EEG and MEG topographies for theta $(\theta)$ power modulations.

$$
\begin{aligned}
& \mathbf{S}=n^{-1} \mathbf{X}_{s} \mathbf{X}_{s}^{T} \\
& \mathbf{R}=n^{-1} \mathbf{X}_{r} \mathbf{X}_{r}^{T}
\end{aligned}
$$

where $n$ is the number of time points minus 1 in $\mathbf{X}_{\mathrm{s}}, \mathbf{X}_{\mathrm{r}}$. A single set of weights (in vector $\mathrm{w}$ ) maximizing the power ratio between $\mathbf{S}$ and $\mathbf{R}$ can be found by maximizing the Rayleigh quotient (Eq. 4), with scalar value $\lambda$ capturing the power ratio (Eq. 5) as follows:

$$
\begin{gathered}
\mathrm{w}=\underset{\mathrm{w}}{\arg \max } \frac{\mathrm{w}^{\mathrm{T}} \mathbf{S} \mathrm{w}}{\mathrm{w}^{\mathrm{T}} \mathbf{R} \mathrm{w}} \\
\lambda=\frac{\mathrm{w}^{\mathrm{T}} \mathbf{S} \mathrm{w}}{\mathrm{w}^{\mathrm{T}} \mathbf{R} \mathrm{w}} \text { at } \mathrm{w}=\mathrm{w}_{\max }
\end{gathered}
$$

Extending the above from a single vector to a matrix computation yields the generalized eigenvalue equation in Equation 6, which, when solved, gives a matrix of weights $\mathbf{W}$ and a diagonal matrix of eigenvalues $\Lambda$ as follows:

$$
\mathbf{R W \Lambda}=\mathbf{S W}
$$

These weights (eigenvectors) capture the directions in which $\mathbf{S}$ and $\mathbf{R}$ are most separable (i.e., maximize the energy ratio between $\mathbf{S}$ and R). The associated eigenvalues capture the energy ratio. The eigenvectors are linearly separable: each eigenvector of length $n$ (provided it is associated with a unique eigenvalue) points in a direction in $n$-dimensional space that maximally separates $\mathbf{S}$ from $\mathbf{R}$, given the constraint that it is distinct from all other eigenvector directions. Notably, $\mathbf{S}$ and $\mathbf{R}$ are symmetric; but because the decomposed matrix is the asymmetric compound matrix $\mathbf{R}^{-1} \mathbf{S}$, the eigenvectors are not constrained to be orthogonal. This is an advantage over PCA, in which the orthogonality constraint limits its usefulness for source separation.

We applied this eigendecomposition procedure to our data. Individual EEG trials (in $\mu \mathrm{V}$ ) and MEG trials (in pT) were $z$-scored to convert the data to a common scale, and combined into a single dataset per subject. Data were bandpass-filtered at theta $(5-7 \mathrm{~Hz})$, and signal matrix $\mathbf{S}$ was computed from a $0-800 \mathrm{~ms}$ time window (see Fig. 2). Reference matrix $\mathbf{R}$ was computed from unfiltered (broadband) data in the same time window, with $1 \%$ shrinkage regularization (Eq. 7) to improve matrix separability as follows:

$$
\begin{gathered}
\widetilde{\mathbf{R}}=(1-\gamma) \mathbf{R}+\gamma \alpha \mathbf{I} \\
\alpha=\frac{1}{\mathrm{n}} \sum_{\mathrm{i}=1}^{\mathrm{n}} \lambda_{\mathrm{i}}
\end{gathered}
$$

where $\tilde{\mathbf{R}}$ is the regularized matrix, $y$ is the degree of shrinkage (here 0.01 ), $\mathbf{I}$ is the identity matrix of equal size to $\mathbf{R}$, and $\alpha$ is the average of the eigenvalues of $\mathbf{R}$.

GED yielded 328 eigenvectors ("components") for each subject, as many as there were sensors. Eigenvectors were normalized to unit length. One subject exhibited a high-energy component that was strongly driven by a single EEG electrode (F4); this electrode was removed from that subject's dataset, and the GED was rerun. Components with repeating eigenvalues (specified as $<1 \%$ difference with the previous largest eigenvalue) were excluded from analysis, as these possibly reflected inseparable components. This rejection affected only a single component with midfrontal topography. Component significance was established using permutation testing, where narrowband-filtered and broadband time series were randomly shuffled into $\mathbf{X}_{\mathrm{r}}$ and $\mathbf{X}_{\mathrm{s}}$ 1000 times to generate a null distribution of eigenvalues (for full procedure, see Hayton et al., 2004). Components explaining significant amounts of variance $(\alpha=0.05)$ were retained for further analysis. 
A

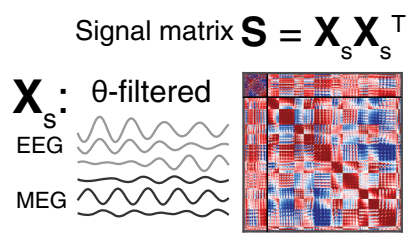

B

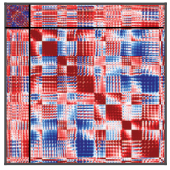

$\mathbf{S}$

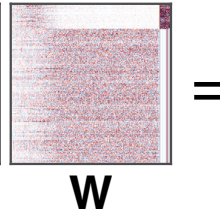

W
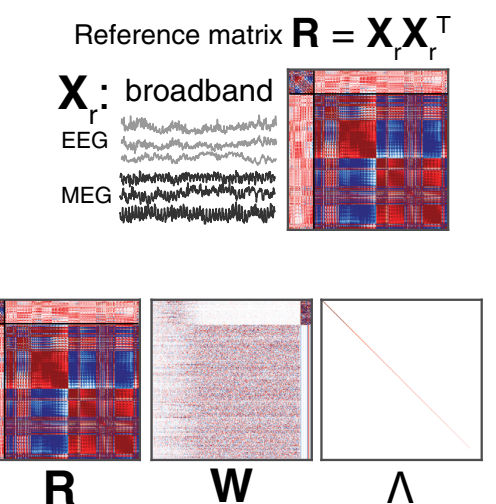

C
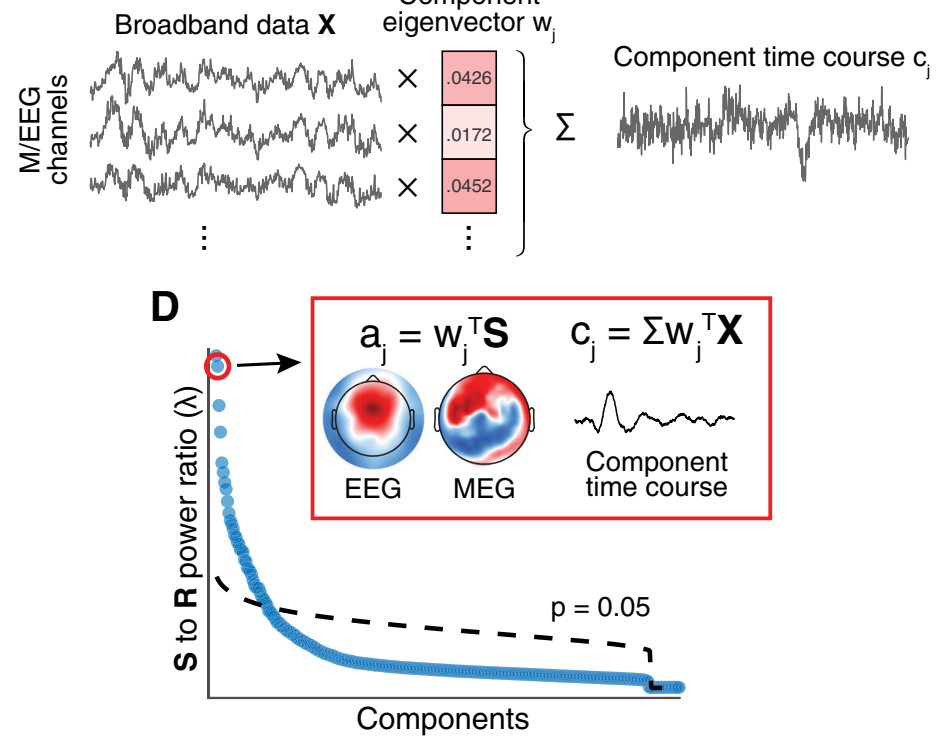

Figure 2. Overview of the source-separation method. A GED was performed for each subject, to identify statistical components contributing to theta-frequency signal. $\boldsymbol{A}$, The signal matrix $S$ is the covariance matrix of all MEG/EEG sensor signals bandpass-filtered at $5-7 \mathrm{~Hz}$, from a $0-800$ ms time window. The reference matrix $\mathrm{R}$ is the covariance matrix of the unfiltered sensor signals from the same time window. The first 56 rows and columns are EEG; the rest are MEG. $\boldsymbol{B}$, The optimal spatial filters for theta-band activity are obtained via the GED equation. $\Lambda$ is a diagonal matrix containing the eigenvalues, and $W$ is the associated eigenvector matrix; matrices are sorted to have the largest components on the left. The larger components mix EEG and MEG weightings, whereas modality-specific filters appear on the right (smaller components). $C$, The canonical time course per trial for each component is constructed as a linear combination of the time series from the $328 \mathrm{MEG} / \mathrm{EEG}$ sensors, using the component eigenvector as weights. $\boldsymbol{D}$, Single-subject eigenspectrum, with significance cutoff $(\alpha=0.05)$ determined by permutation testing. Inset, EEG and MEG topographies derived from the spatial filter, and the trial-averaged component time course (ERP).

A component topography $a_{j}$ was computed for each given eigenvector $\mathrm{w}_{\mathrm{j}}$ (Eq. 9, also shown in Fig. 2D) (compare Haufe et al., 2014). Topographies were computed separately for EEG and MEG to ensure that topographies remained modality-specific and unaffected by crosscovariances between EEG and MEG channels as follows:

$$
a_{j}=w_{j}^{T} S
$$

Furthermore, component time-series $c_{j}$ were constructed for each trial (also shown in Fig. 2D) as follows:

$$
c_{j}=w_{j}^{T} \mathbf{X}
$$

The component topography was used for topographical interpretation, and the component time series was used in all time-series analyses, described later.
Selecting midfrontal sources. Response conflict theta manifests with stereotypical midfrontal topography on EEG recordings. To select components with midfrontal topographies, we constructed a topographical EEG template (see Fig. 4) that consisted of a Gaussian centered on midfrontal electrode $\mathrm{FCz}$, where conflict theta power is typically maximal. Shared spatial variances $\left(R^{2}\right)$ between component EEG topographies and the midfrontal EEG template were calculated. We visually inspected the topographies and imposed a cutoff, retaining components with $R^{2}>0.5$. As eigendecomposition returns eigenvectors without a canonical sign, we inverted EEG topographies so that they correlated positively with the midfrontal template, thus representing the typical conflict-related power increase over midfrontal electrodes. Furthermore, we inverted MEG topographies so that they were positive at the right lateral set of sensors. Sign-flipping facilitates cross-subject averaging and visual interpretation of topographies but does not change the timefrequency response of the component time series.

Confirming source robustness using ICA. To confirm that our main observation (multiple theta sources) was independent of the exact source separation method used, we applied the JADE algorithm for ICA to the theta band-filtered data. Source separation was limited to 60 independent components (ICs) per subject to prevent the ICA from fitting noise. This number was chosen based on the maximum number of significant GED components (46) across subjects, plus some leeway to allow for variability in the ordering of ICs.

Confirming source robustness using splithalf validation. We validated within-subject source stability by performing a split-half component validation procedure, following the method suggested by Groppe et al. (2009) with changes as appropriate. Briefly, components obtained from the full dataset were greedily paired to components obtained from two split-halves consisting of alternating trials, by comparing their Euclidean topographical distances. Next, each triplet's significance was computed using the pooled topographical distances across subjects as the null distribution. Triplets with significantly low topographical distances $(p<0.05)$ were taken to be stable components.

We made three notable changes to the method laid out by Groppe et al. (2009). First, we constrained our validation procedure to the top 60 components from the split-halves and to the significant components from the full set, to limit spurious matches arising from pairwise comparisons among 328 components. Second, we computed the topographical distance function differently (separately for EEG and for MEG, then averaged together) to prevent bias toward MEG on account of the larger number of sensors. Third, we omitted the time-series distance metric altogether and instead used the all-to-all topographical distances for the null distribution. This was in part because component time series and component topographies comprise the same linear weighting of different data and should have proportional pairwise distances, and in part because computing $3 \times 328 \times 328$ time series and the pairwise distances between them was computationally infeasible.

Confirming source multimodality. Inspired by the observation that many components had similar EEG topographies but variable MEG 
topographies, we investigated the possibility that the multimodality of our observed components could have been an artifact of the GED algorithm.

First, we quantified the degree to which our observed eigenvectors mixed EEG and MEG sensor weights, and compared the degree of mixing for significant versus nonsignificant eigenvectors. A modality dominance (MD) value was computed for each component as follows:

$$
\mathrm{MD}=\left(\frac{\mathrm{RMS}_{\mathrm{EEG}}-\mathrm{RMS}_{\mathrm{MEG}}}{\mathrm{RMS}_{\mathrm{EEG}}+\mathrm{RMS}_{\mathrm{MEG}}}\right)^{2}
$$

where RMS $\mathrm{EEG}_{\text {and }}$ RMS $\mathrm{MEG}$ are the root mean square of the EEG sensor weights and the MEG sensor weights, respectively. An MD value of 0 means that the energy of the sensor weights is completely balanced, and an MD value of 1 means that one of the modalities completely dominates the component. To determine whether the observed multimodality in our components was a property of the components or of the GED procedure, MD values were compared for significant components (capturing theta-related variance) and nonsignificant components (capturing non-theta-related variance).

Next, we performed a single-subject control analysis (on S03) in which we nullified the crossmodal covariances between EEG and MEG, rendering the data unimodal. This was achieved by simply setting the cross-terms in covariance matrices $\mathbf{S}$ and $\mathbf{R}$ to zero. A GED was then performed on the unimodal covariance matrices, and an MD value was computed for each of the resulting eigenvectors (Eq. 11). If the GED procedure respects the unimodality of the data, the identified eigenvectors should also be purely unimodal and have MD values of 1 .

Evaluating source similarity. To address the possibility that GED artificially split a single source into multiple components, we evaluated components on three measures of signal similarity. In preparation, theta power (relative to a -500 to $-100 \mathrm{~ms}$ baseline) and phase were extracted from the component time series, through convolution with a $6 \mathrm{~Hz}$ complex Morlet wavelet. For the first measure, pairwise component synchrony was computed as the mean length of the phase angle difference vector at each time point, and mean synchrony over -500 to $900 \mathrm{~ms}$ was extracted. This measure captured the component phase similarity. Synchrony between adjacent EEG electrodes $\mathrm{Pz}$ and $\mathrm{POz}$ (where synchronization was expected to be inflated by volume conduction) was computed for comparison. Second, the mean pairwise correlation between component theta power time courses was computed, capturing the similarity of within-trial power fluctuations. Third, the pairwise correlation between mean component theta power over $0-800 \mathrm{~ms}$ was computed, capturing the similarity of power fluctuations across trials.

Another possibility is that a single midfrontal theta source was split into two components because of noise. To investigate this possibility, we determined how much noise needed to be added to two copies of a single spatial filter to generate synchronization of a similar magnitude as two empirical spatial filters. This was a three-step process. First, we selected a spatial filter (the eigenvector w from Eq. 4), made a copy of this filter, and then added unique white noise to each copy (noise $\mathrm{SD}, \mathrm{SD}_{\text {noise, }}$ ranging from 0 to 10 times the eigenvector $\mathrm{SD}, \mathrm{SD}_{\text {eig }}$, in steps of $0.1 ; 50$ copies per noise level). Second, theta power time courses were derived from these noisy spatial filters. Third, the expected distribution of pairwise correlations between time courses was computed for each of the noise levels (a total of $1 / 2 \times 50 \times 50-50=1200$ unique pairwise "noisy self-correlations" per noise level). One-tailed $t$ tests ( $\alpha=0.05$, no multiple comparisons correction) between the correlation distributions determined the noise level for which the generated self-correlations were no longer statistically distinguishable (for at least 1 participant) from the empirical cross-component correlations.

Component-level time-frequency analysis. The retained component time series were time-frequency decomposed through trial $\times$ trial convolution with 40 complex Morlet wavelets, as previously described for data from sensor FCz. The average time-frequency spectrum across components was computed. To accurately represent each component's contribution to the overall energy, each component was weighted by the variance it explained among the retained components per subject.
Computing Granger causality (GC). To attempt to identify a hierarchy in how components interacted over time, we computed windowed GC on component time series (see Fig. 7A) using the MATLAB MVGC toolbox (Barnett and Seth, 2014). The ERP over trials was subtracted from each trial to limit the influence of nonstationarities. Data were downsampled from 1200 to $300 \mathrm{~Hz}$. Model order was estimated per subject, on all trials, over $0-800 \mathrm{~ms}$, using Bayes' information criterion. We selected the maximum order across subjects (6 time steps, equaling $19.2 \mathrm{~ms}$ ) for all further GC calculations. Next, trial data were segmented into $200 \mathrm{~ms}$ windows with $50 \mathrm{~ms}$ step size. Conditional GC between each pair of components was computed over each time window as follows:

$$
\mathrm{GC}_{\mathrm{j} \rightarrow \mathrm{i} \mid \mathrm{k}}=\ln \frac{\Sigma_{1}}{\Sigma_{2}}
$$

where $j$ is the driving component, $i$ is the receiving component, and $k$ is the group of all other components, on which $j \rightarrow i$ is conditional. $\Sigma_{1}$ is the error term (variance of the residual) of the autoregressive model fit to the recent history (constrained by the order parameter) of $i$, with linear terms for the influence of the components in $k . \Sigma_{2}$ is the error term for the same model with the addition of a linear term for the influence of $j$. Thus, if the prediction for $i$ improves with incorporating the past of $j$, the GC value exceeds 0. Averaging over "outgoing" and "incoming" GC values yielded a "driving mass" (DM) and "receiving mass" (RM) value for each component within each time window:

$$
\begin{aligned}
& \mathrm{DM}_{\mathrm{j}}=\frac{1}{\mathrm{n}-1} \sum_{\mathrm{i} \neq \mathrm{j}}^{\mathrm{n}} \mathrm{GC}_{\mathrm{j} \rightarrow \mathrm{i} \mid \mathrm{k}} \\
& \mathrm{RM}_{\mathrm{j}}=\frac{1}{\mathrm{n}-1} \sum_{\mathrm{i} \neq \mathrm{j}}^{\mathrm{n}} \mathrm{GC}_{\mathrm{i} \rightarrow \mathrm{j} \mid \mathrm{k}}
\end{aligned}
$$

where $n$ is the number of components for that subject. Driving and receiving mass were computed separately for incongruent trials and congruent trials, with condition-average GC being the average of these two.

Deriving task and conflict modulation. For each component, theta power modulation during the task was computed as mean theta power on all trials (normalized relative to a -500 to $-100 \mathrm{~ms}$ baseline) over $0-800 \mathrm{~ms}$. This yielded a ratio (i.e., a modulation value of 1 indicated no change in theta power compared with baseline). Conflict-related modulation was computed as mean theta power modulation on incongruent trials minus modulation on congruent trials. These values were also computed at midfrontal electrode $\mathrm{FCz}$, for comparison purposes. One component was rejected for being strongly negatively task-modulated, leaving 52 components. Component-specific GC modulation was computed in the same way as theta power modulation: once across all task conditions and once per condition, although computed over driving and receiving mass instead of theta power.

Extracting temporal and spatial component features. We applied PCAs to identify temporal and spatial features of the component time courses and topographies that were consistent across subjects. Separate PCAs were applied to EEG topographies, MEG topographies, theta power time courses, and GC time courses. The number of PCs for each data feature was determined by visually identifying the "elbow" in the plot of the eigenvalues (i.e., the point where additional explained variance per PC sharply decreases) and retaining the PCs before that point. This approach suggested two PCs for each data feature. As PCA returns PCs without a canonical sign, we inverted the time course PCs and topography PCs so that they were positively correlated with the majority of original time courses and topographies.

Statistical analysis. Statistical analyses were performed using MATLAB (MATLAB 2014a, The MathWorks). The significance level was set at $\alpha=0.05$. Two-sample differences were compared using oneway or two-way $t$ tests (described in Results), with Benjamini-Hochberg step-up multiple comparisons correction (Benjamini and Hochberg, 1995) applied when testing related hypotheses. Behavioral results were 
A

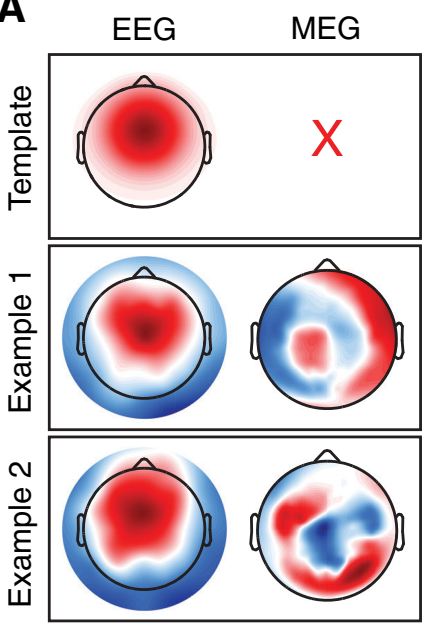

C
B

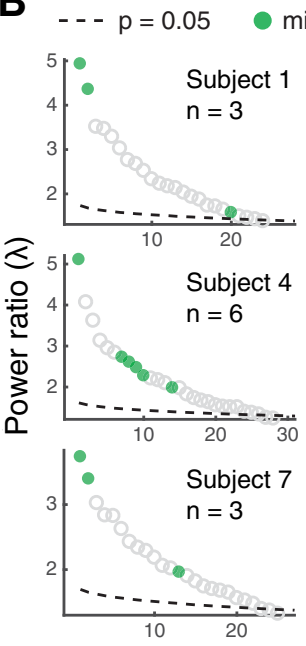

midfrontal $\left(R^{2}>0.5\right)$

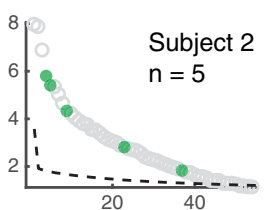

not midfrontal $\left(R^{2} \leq 0.5\right)$
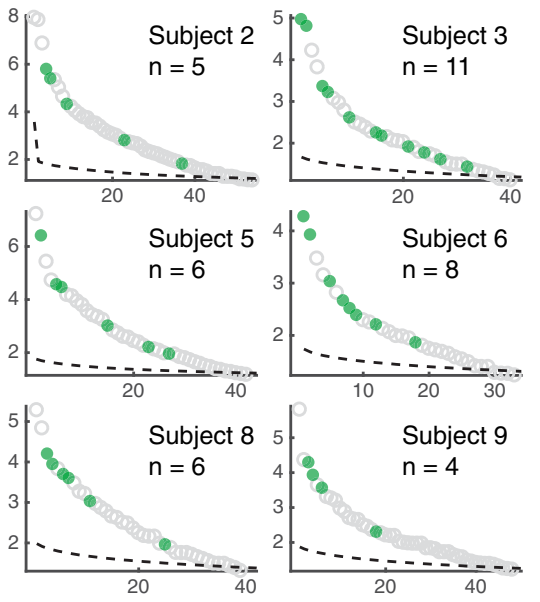

Component

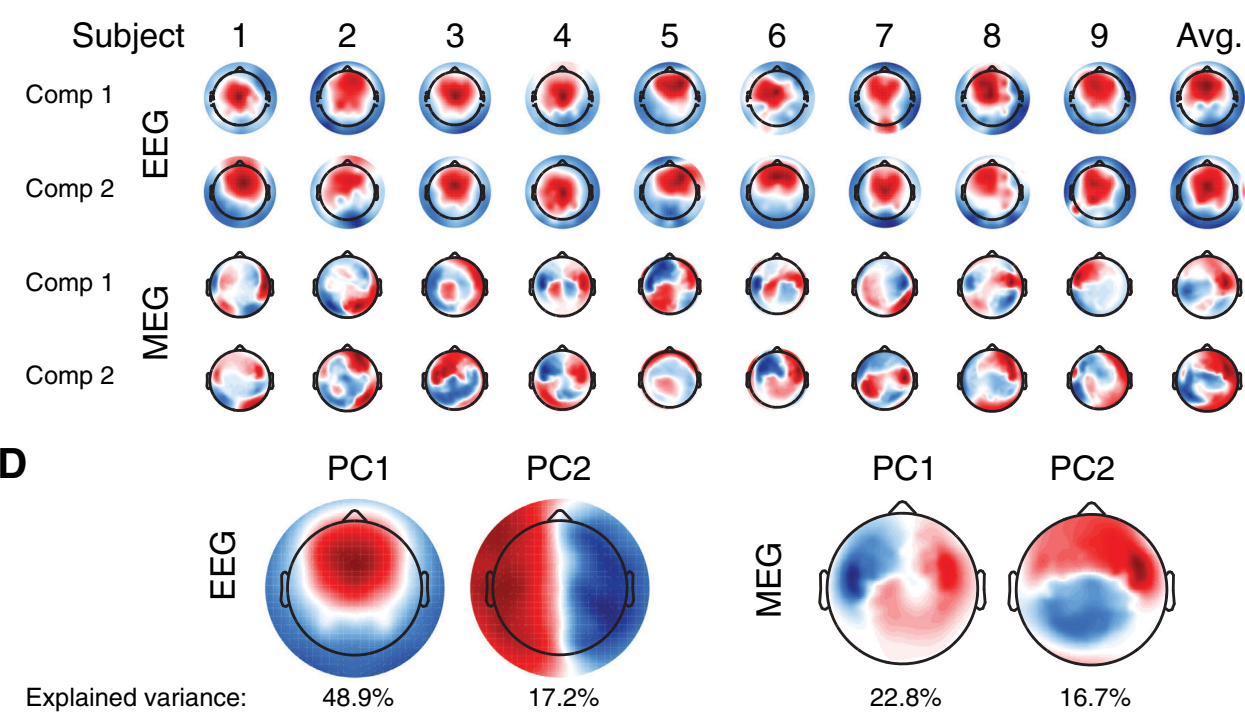

Figure 3. Procedure for selecting midfrontal theta components based on eigenvalue statistical significance and EEG topography template matching. $A$, Top, The EEG template topography used to select midfrontal components. Middle and bottom, Components selected using this template can have diverse MEG topographies. $B$, Components sufficiently $\left(R^{2}>0.5\right)$ correlated with the template were retained (green). $n$ indicates the number of components retained per subject. Most subjects had at least two midfrontal components in their top 5 theta components, indicating that our conclusion that midfrontal theta is driven by more than one component is not dependent on the particulars of significance thresholding. Components were selected based on theta energy (reflected in the power ratio) and midfrontal EEG topography; possible modulation by experiment condition was ignored during selection. C, The two top midfrontal components (explaining most variance) per subject exhibited homogeneous EEG topographies and heterogeneous MEG topographies. D, PCAs individually applied to the EEG and MEG topographies captured salient spatial features across all components.

compared using a two-way ANOVA to identify interactions, with Benjamini-Hochberg multiple comparisons correction applied to prechosen contrasts. For GC marginals, tested at each time window and for each pairwise combination of components, we applied false discovery rate multiple comparisons correction as implemented in the MVGC toolbox (Barnett and Seth, 2014). No multiple comparisons corrections were applied when determining at which noise level the randomized and empirical component correlation coefficients became indistinguishable (see Fig. 5). This lack of multiple comparisons correction is intentional so that the identified noise level represents a lower bound. Component significance was determined by creating a null distribution using permutation testing, with a cutoff for retention set at $p=0.05$. Correlation coefficients were computed as Pearson's $r$. Descriptive statistics are always given as mean $\pm \mathrm{SD}$.

Code and data accessibility. The custom-written MATLAB analysis code is available on GitHub at http://www.github.com/marrit-git/ MEEG-multiple-theta-sources. The MEG/EEG data are available from https://data.donders.ru.nl.

\section{Results}

Behavioral results

We replicated the typical congruency sequence effect (also known as the "Gratton effect") of previous trial congruence affecting performance on the next trial (Gratton et al., 1992). Following congruent trials, accuracy was $94.7 \pm 3.1 \%$ on congruent and $86.5 \pm 7.1 \%$ on incongruent trials, whereas following incongruent trials, accuracy was $91.2 \pm 3.7 \%$ on congruent and $92.0 \pm 6.0 \%$ on incongruent trials (interaction between previous and current congruency: $p=0.015, F_{(1,32)}=6.64$, ANOVA). This effect was also observed in reaction times (Fig. $1 B$ ): subjects responded faster on incongruent trials following incongruent $(465.9 \pm 22.5 \mathrm{~ms})$ than congruent $(489.6 \pm 26.5 \mathrm{~ms})$ trials, and responded slower on congruent trials following incongruent $(477.8 \pm 31.6 \mathrm{~ms})$ than congruent $(444.7 \pm$ $35.8 \mathrm{~ms}$ ) trials (interaction $p=0.007, F_{(1,32)}=8.30$, ANOVA). As mentioned in Materials and Methods, we focused the 
MEG/EEG analyses on trials following congruent trials, to maximize the conflict effect.

Sensor-level analyses

Data recorded at midfrontal EEG electrode $\mathrm{FCz}$ were timefrequency decomposed through complex Morlet wavelet convolution. This decomposition served two purposes. The first was to confirm that task and conflict effects were present in the sensorlevel data, rather than being artificially induced by the GED. The second was to guide the feature selection for the GED.

The average $\mathrm{FCz}$ time-frequency spectra (Fig. 1C) exhibited a task-related theta power increase, as well as conflict modulation in the theta band. Power increased predominantly within the 4$7 \mathrm{~Hz}$ frequency range. This observation validated our choice of bandpass frequency filter $(5-7 \mathrm{~Hz}$, chosen for a priori expectations of theta manifesting at $\sim 6 \mathrm{~Hz}$ ) for the GED signal matrix. The condition-average theta power increase spanned $0-800 \mathrm{~ms}$ relative to stimulus onset, guiding our selection of this time window to construct the GED signal and reference matrices.

Condition-averaged theta power (at $0-800 \mathrm{~ms}$ relative to a -500 to $-100 \mathrm{~ms}$ baseline) was observed at midfrontal and lateral occipital locations in EEG, and at midfrontal and frontolateral locations in MEG (Fig. 1D). Conflict-modulated theta power was maximal at midfrontal locations in EEG and was broadly midfrontally distributed in MEG.

Theta power at $\mathrm{FCz}$ was significantly greater during the task $(1.38 \pm 0.68 \mathrm{~dB})$ than during baseline $(p=0.0001$, one-sided $\left.t_{(8)}=6.08\right)$. However, $\mathrm{FCz}$ theta power was not significantly greater on incongruent $(1.46 \pm 0.78 \mathrm{~dB})$ than congruent trials in the window of $0-800 \mathrm{~ms}(1.28 \pm 0.65 \mathrm{~dB} ; p=0.14$, one-sided $t_{(8)}=1.15$; all Benjamini-Hochberg-corrected).

\section{Identification of midfrontal theta components}

Custom spatial filters were created using GED, to identify components in the data that maximally separated theta-band activity from broadband data (procedure illustrated in Fig. 2). A permutation testing-determined significance threshold ( $\alpha=0.05$ ) was applied to the resulting 328 components per participant (Fig. 2D). As shown in Figure 3, each subject exhibited between 21 and 46 significant theta components (average 33.89 \pm 9.47 ). A subset of these $(5.89 \pm 2.47$ per subject; minimum 3 , maximum 11) was midfrontal, defined as shared spatial variance $R^{2}>0.5$ between the component's EEG topography and the midfrontal template shown in Figure $3 \mathrm{~A}$. By virtue of the template-based selection method, component EEG topographies were homogeneous. However, the associated MEG topographies were highly variable (Fig. 3C). This variability is also reflected in the salient topographical features identified by applying PCAs to the EEG and MEG topographies (Fig. $3 D$ ): a single PC explained $48.9 \%$ of the variance in the EEG topographies, whereas the top two MEG PCs together captured $39.5 \%$ of the variance.

Averaged time-frequency decompositions of the retained components (Fig. 4) were similar to those at electrode FCz (Fig. $1 C)$. This similarity suggests that this subset of the data retained the salient data features, and that applying GED had no distorting effects.

\section{Alternative source separation using ICA}

We confirmed that the detection of multiple midfrontal theta components was not dependent on the source separation method by using ICA to separate 60 ICs per subject in the theta band-filtered data. Of these 60 ICs, an average of 6.33 ICs per subject ( \pm 2.95 , minimum 4, maximum 13) had midfrontal

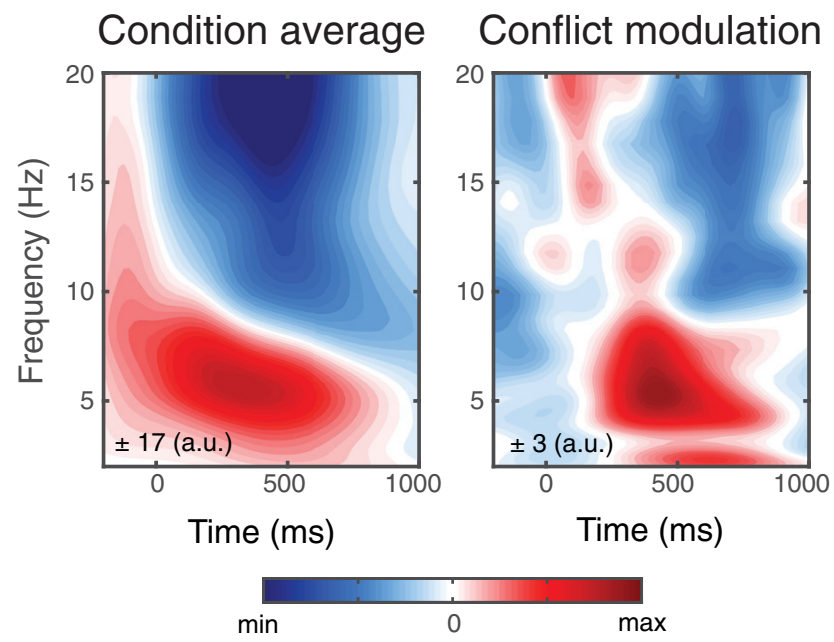

Figure 4. Average midfrontal component time-frequency decompositions appear qualitatively similar to those from electrode FCz (in Fig. 10). Components were weighted by their relative eigenvalue (relative to all within-subject midfrontal components), as a measure of their contribution to the overall signal.

topographies according to our criteria. This is consistent with previous reports of multiple frontal theta ICs within-subject (Töllner et al., 2017). As GED is both spectrally specific and more amenable to inferential statistics, we focused on GED and did not further consider the ICs.

\section{Within-subject component robustness}

We performed split-half validation of each subject's components, by pairing triplets of components from the full set and two splithalves of the data. Triplet significance was computed using the pooled topographical distances over subjects as the null distribution. For all subjects, multiple significant midfrontal full-set components $(5.11 \pm 2.62$, minimum 2 , maximum 10$)$ were stable across both halves (i.e., had significantly low topographical distances to the full-set component, both $p<0.05)$.

The majority of midfrontal components $(86.6 \pm 19.7 \%$ of the originals) were thus robust against subsampling of the data. As we cannot discern whether the remaining components were truly spurious, were lost because of variable component ordering, or became nonsignificant because of the reduced signal-to-noise ratio from using only half the data, we continued our analyses on all of the significant components found in the full set.

\section{Component multimodality}

Midfrontal theta components were characterized by spatial filters that captured both EEG and MEG activity. We evaluated whether this multimodality was a property of the components or caused by the GED procedure. An MD value, ranging from 0 (perfectly balanced) to 1 (perfectly unimodal), was computed for each component eigenvector (Eq. 11). MD values were compared for significant components, which capture theta-related variance and presumably have meaningful sensor weights, and nonsignificant components, which capture non-theta-related variance. When pooled across subjects, significant components had lower MD values $(0.16 \pm 0.13)$, indicating more balanced contributions from MEG and EEG compared with the nonsignificant components $\left(0.32 \pm 0.22, p<0.0001\right.$, one-tailed $\left.t_{(2927)}=11.5\right)$. Withinsubject comparisons (with lower statistical power) showed significantly lower MD values for significant than nonsignificant components for 4 of 9 subjects ( $p<0.05$, one-tailed $t$ test, variable $t$, variable df, all Benjamini-Hochberg-corrected). From 

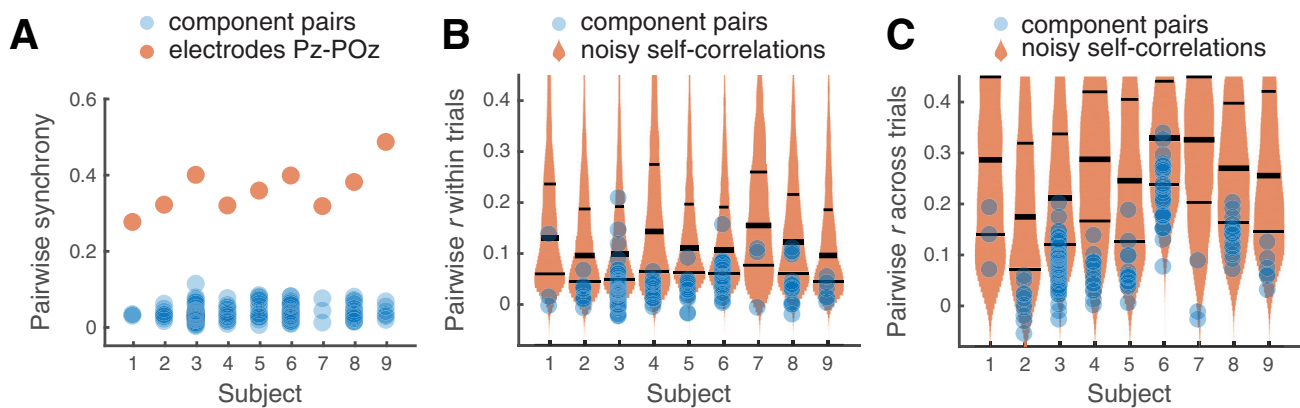

Figure 5. Several control analyses provided evidence that the components reflected unique data features, as opposed to single theta sources artificially split into multiple components. $\boldsymbol{A}$, Pairwise synchrony across components (calculated as the mean length across trials of the phase difference vector) was generally weak. For comparison, orange dots represent synchronization between $\mathrm{Pz}$ and $\mathrm{POz}$, where the interelectrode synchronization can be expected to be inflated by volume conduction. $\boldsymbol{B}$, Trial $\times$ trial correlations between theta power time series for different components were generally low (blue dots). Orange violin plots represent distributions of correlations between copies of the same component but with white noise added. At SD ${ }_{\text {noise }}=1.9 \times$ $S D_{\text {eig, }}$ generated correlations first became statistically indistinguishable ( $p>0.05$ for at least 1 subject) from the empirical pairwise component correlations. This suggests that the GED-identified components were unlikely to originate from shared theta sources in the presence of only modest levels of noise. Horizontal lines in the violin plots indicate mean \pm SD. $C$, Similar to $\boldsymbol{B}$, but for mean theta power per trial, and with the orange violin plots representing the distribution of correlations for $S D_{\text {noise }}=1.5 \times S D_{\text {eig }}$ (at which generated and empirical correlations first became indistinguishable).

these results, we concluded that the mixing of EEG and MEG sensor weights was a genuine property of the theta components.

We additionally performed a control analysis to verify that the GED procedure respected data unimodality. Unimodal covariance matrices were created by zeroing the crossmodal coefficients in matrices $\mathbf{S}$ and $\mathbf{R}$, and a GED was applied to these matrices. MD values were computed for the resulting eigenvectors. As expected, the MD value for each of these eigenvectors was 1 , indicating that GED correctly identified only modalityspecific spatial filters in the absence of crossmodal covariances and did not introduce any additional mixing. We concluded that the observed mixing of EEG and MEG weights in the larger GED components represented a true coupling between EEG- and MEG-measured brain dynamics.

Possible alternative accounts for multiple theta components To rule out the possibility that the GED artificially split out a single signal source into multiple components, we computed three measures of component similarity, reasoning that components representing the same source should have highly similar time courses.

The first measure, pairwise synchrony at $6 \mathrm{~Hz}$, was generally low (Fig. 5A; $0.044 \pm 0.005$ ) compared with synchronization between EEG electrodes $\mathrm{Pz}$ and $\mathrm{POz}$, which will be artificially inflated because of volume conduction contamination. These electrodes were significantly more synchronous than any pair of components $\left(0.360 \pm 0.062, p<0.001\right.$, two-sided $\left.t_{(16)}=15.2\right)$. As such, components did not exhibit high similarity in terms of phase.

The second measure, trial-averaged pairwise correlations between theta power time courses, was also generally low (Fig. $5 B$; Pearson's $r=0.04 \pm 0.02$ ), indicating low similarity of within-trial power fluctuations. The third measure, pairwise correlations between mean theta power on each trial, was likewise low (Fig. 5C; Pearson's $r=0.11 \pm 0.08$ ).

Another potential explanation for observing multiple theta components is that noisy data caused a single real source to be split into multiple apparent sources. To determine the feasibility of this explanation, we created two noisy copies of each identified spatial filter, computed their component time series, and correlated these with each other. We then increased the amount of noise until the noise-driven time-series correlations were no longer statistically distinguishable from the empirical time-series correlations. We found that a noise factor of $\mathrm{SD}_{\text {noise }}=1.9 \times \mathrm{SD}_{\text {eig }}$ for within-trial correlations, and $\mathrm{SD}_{\text {noise }}=1.5 \times \mathrm{SD}_{\text {eig }}$ for crosstrial correlations, was required to reach this point (lower bound on noise; reported $\mathrm{SD}_{\text {noise }}$ measured at the first occurrence across subjects of $p>0.05$ between generated and empirical correlation distributions; one-tailed $t<1.55$ within-trial, one-tailed $t<1.52$ cross-trial, varying df; no multiple comparisons correction). In other words, if components comprised noisy representations of the same source, the noise would have to exceed the signal by $\sim 2$ SDs to generate comparably low pairwise correlations.

Finally, we tested whether multiple statistical sources may have arisen from a single real source that changed over time (e.g., with head movement or changes in signal quality). We reasoned that this account would predict negatively correlated cross-trial power fluctuations. Pairwise correlations across trials were not significantly negative $\left(p>0.99\right.$, one-sided $t_{(8)}=3.93$ against $\mu=0$ ), suggesting that components were stable over time.

Task and conflict modulation of theta power

To characterize the nature of these components, we extracted the theta power time courses from the component time series. We then quantified how theta power changed during the task (relative to baseline) and how it changed on incongruent versus congruent trials. Results are shown in Figure 6. Component theta power was significantly greater during the task $(1.04 \pm 1.13 \mathrm{~dB})$ than during baseline $\left(p<0.0001\right.$, one-sided $\left.t_{(51)}=6.67\right)$. Component theta power was significantly greater on incongruent trials $(1.10 \pm 1.19 \mathrm{~dB})$ than on congruent trials $(0.97 \pm 1.09 \mathrm{~dB}$, $p=0.0012$, one-sided $t_{(51)}=3.20$; all Benjamini-Hochbergcorrected).

Because the GED identified linearly separable components, simple averaging may fail to identify meaningful cross-component functional variability. We therefore applied a PCA to the component's theta power time courses (Fig. 6D), which identified a later peak (Fig. $6 E, \mathrm{PC} 1$; peaking at $\sim 480 \mathrm{~ms}$ after stimulus) and an earlier peak (PC2; peaking at $\sim 180 \mathrm{~ms}$ after stimulus) in theta power. Prestimulus effects are attributable to temporal smoothing from the wavelet convolution.

GC

Response conflict-related midfrontal theta is classically considered to be a phenomenon with a singular origin. While our 
A

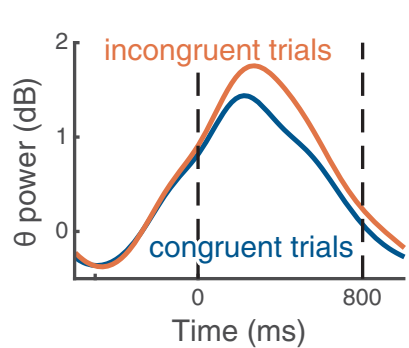

D

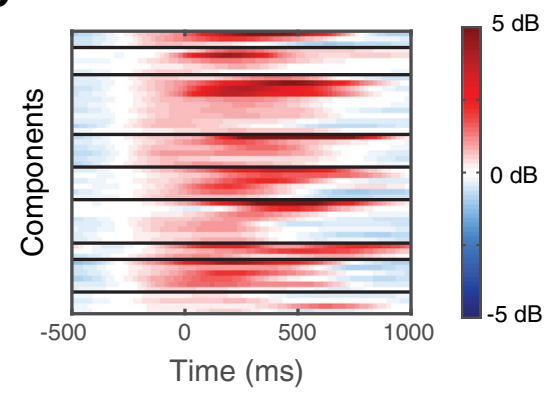

B

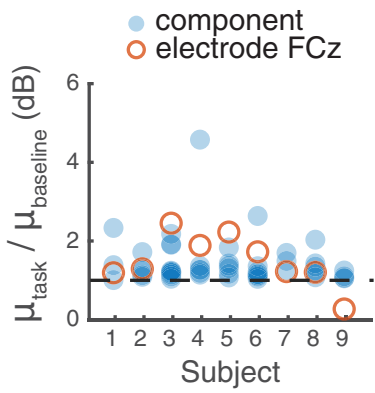

C

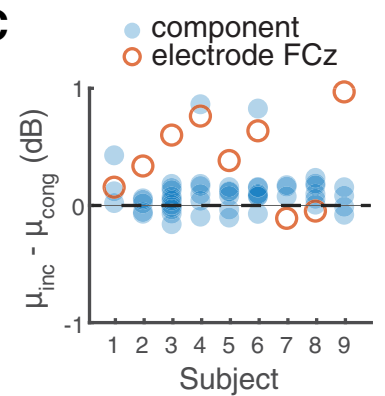

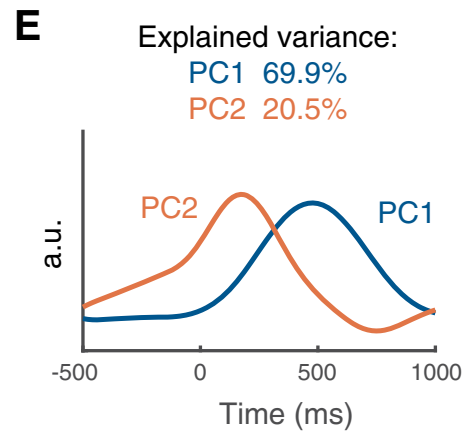

Figure 6. Characterization of component theta $(\theta)$ power dynamics over time and conflict conditions. $\boldsymbol{A}$, Component-averaged theta power per condition. $\boldsymbol{B}$, Mean theta power modulation during the task $(0-800 \mathrm{~ms})$ relative to baseline $(-500$ to $-100 \mathrm{~ms})$ for each component (blue dots) and electrode $\mathrm{FCz}$ (orange circles) for each subject. $C$, Similar to $B$, but showing theta power modulation during incongruent compared with congruent trials. $\boldsymbol{D}$, Components exhibited variability in theta power over time. Black lines separate components originating from different subjects. $\boldsymbol{E}, \mathrm{PCA}$ revealed salient temporal features of the theta power time series in $\boldsymbol{D}$. The top PC reflects a later theta power increase, explaining most of the variance, and the second PC reflects an earlier theta power increase.

previous results suggest that this is not the case, we considered the related possibility that activity from one of the identified components dominated the other components, which would lend credence to the singular-origin interpretation. To address this question, we computed GC over time between components, quantifying the extent to which component time series predicted one another. All-to-one connectivity (RM) and one-toall connectivity (DM) were determined for each component. $\mathrm{DM}$ and RM were significant $(p<0.05$ for all components and at all time points, $\chi^{2}$ test, false discovery rate multiple comparisons corrected), as were virtually all pairwise connections at each time point, suggesting that components exchanged information. Baseline-normalized time courses are shown in Figure $7 B$.

We first investigated whether this information exchange was modulated by task conditions. Average component DM did not significantly differ between the task $(2.43 \pm 1.12$, all GC values in this section $\left.\times 10^{-3}\right)$ and baseline $(2.59 \pm 1.24 ; p=0.014$, Benjamini-Hochberg-corrected $\alpha=0.0125$, two-sided $t_{(51)}=$ 2.53). The same was true for RM (task $2.39 \pm 1.11$, baseline $2.55 \pm 1.30, p=0.08$, two-sided $\left.t_{(51)}=1.79\right)$. Similarly, component DM did not significantly differ between incongruent $(2.46 \pm 1.24)$ and congruent trials $(2.39 \pm 1.16 ; p=0.11$, twosided $t_{(51)}=1.60$ ), and the same was true for RM (incongruent $2.42 \pm 1.18$, congruent $2.37 \pm 1.05, p=0.16$, two-sided $t_{(51)}=$ 1.42; all Benjamini-Hochberg-corrected). Information transfer thus remained stable on average, regardless of task features.

Next, we determined how information transfer was distributed across individual components. If a single component per subject drove the other components, we would expect to observe (1) that one component especially strongly predicts information in the other component time series, leading to increased DM for that component, and (2) that one component is especially weakly predicted by the other components, leading to decreased RM for that component. The actual per-component DM and RM, shown in Figure $7 C$, do not match either of these expectations. Additionally, DM and RM time series per component (Fig. 7D) were highly correlated $(r=0.79, p<0.001)$, suggesting strong symmetry in components' driving and receiving tendencies.

PCAs applied to the DM and RM time courses (shown in Fig. $7 D$ ) revealed salient temporal features (Fig. 7E). The first PC for both DM and RM captured a slow decrease in GC. The second PC captured a sharper increase, followed by a decrease, of GC.

\section{Discussion}

In this study, we tested the assumption that midfrontal conflict theta is a unidimensional phenomenon (Cavanagh et al., 2012; Cavanagh and Frank, 2014; Cohen, 2014a). We used featureguided source separation to attempt to identify multivariate components contributing to midfrontal theta. All subjects exhibited multiple $(5.89 \pm 2.47)$ midfrontal components that contributed to increases in theta power during a response conflict task. The identified midfrontal components explained significant amounts of variance in the data, reflected unique data features, and remained stable over time. Thus, conflict-related midfrontal theta exists in a high-dimensional signal space, whose basis vectors are sufficiently similar that they appear as a single dimension without careful inspection and multivariate analysis methods (Fig. 8). This multidimensionality suggests that the midfrontal conflict theta signal consists of the aggregate activity of many, diverse, theta generators.

GED, the feature-guided source separation method used here, has several advantages over "blind" source separation methods, such as PCA and ICA. Whereas PCA and ICA optimize for sources that best describe the entire dataset, GED optimizes for sources that maximally separate two specified datasets, effectively maximizing the signal-to-noise ratio (de Cheveigné and Parra, 
A

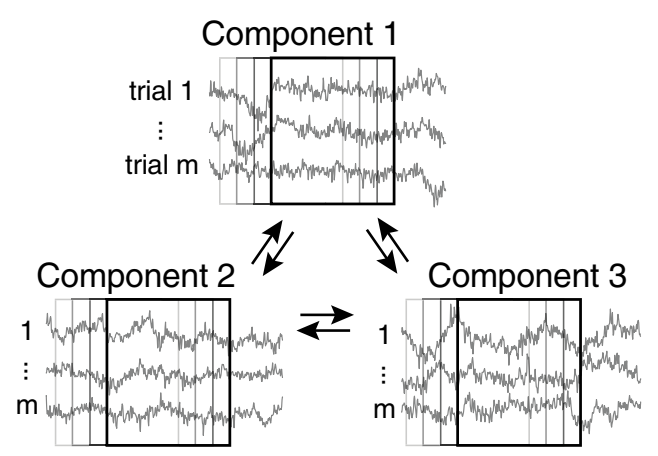

B
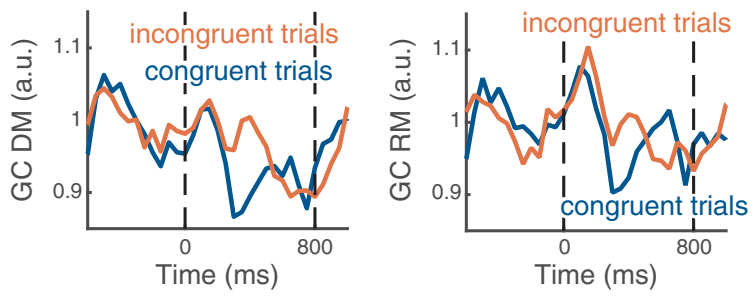

C

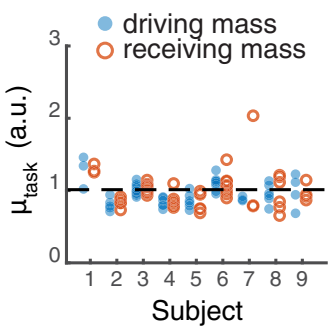

D

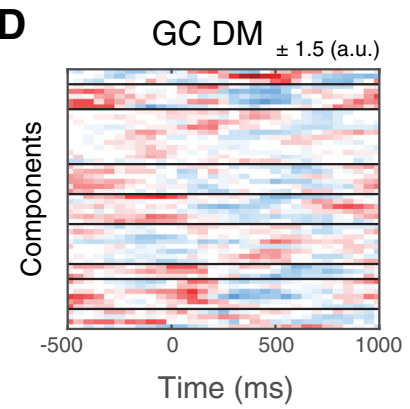

E

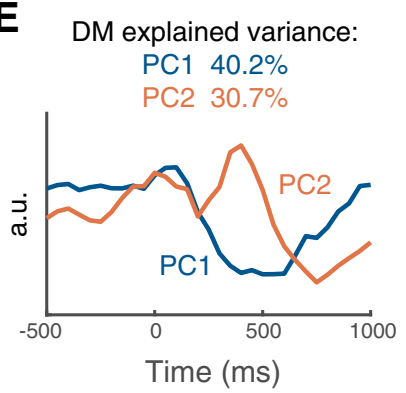

A

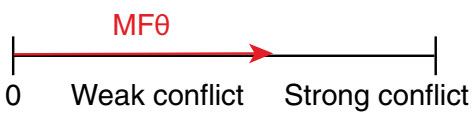

B

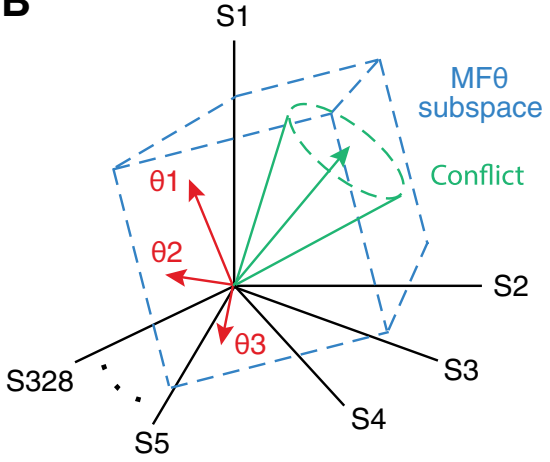

Figure 8. Conceptual visualization of midfrontal theta as a unidimensional versus a multidimensional phenomenon. $\boldsymbol{A}$, In the classical view, response conflict theta is a singular phenomenon that varies in magnitude with the level of conflict. $\boldsymbol{B}$, Our results underpin a characterization of midfrontal conflict theta as occupying a multidimensional subspace within sensor space (sensors S1-S328). The independent variability of the basis vectors (shown as theta components $\theta 1-\theta 3$ ) implies the existence of multiple independent generators of midfrontal theta, a subset of which are conflict-relevant. As conflict increases, theta activity increases, and the signal state moves further away from the origin.

2014). Thus, given a justified a priori selection of data features of interest, GED can achieve higher sensitivity to relevant data features. This sensitivity may explain discrepancies between the current work and the study by Töllner et al. (2017), which used ICA to identify midfrontal theta generators. Töllner et al. (2017) described two frontal midline theta clusters spanning multiple subjects. One cluster was localized to MFC and generated more theta-frequency activity in the presence of conflict; the other was localized to the more anterior $\mathrm{MPFC}$ and was unmodulated by conflict. Importantly, these clusters manifested with dissociable EEG topographies. We here report multiple components per subject with highly similar EEG topographies (e.g., Fig. 3), selected using an FCz-centric template that corresponded closely to the MFC cluster topography found by Töllner et al. (2017). Furthermore, Figure $6 C$ shows that a majority of the components we identified generated more theta-frequency activity in the presence of conflict. In contrast, Töllner et al. (2017) reported at most a single IC per subject that was modulated by conflict. Given the sensitivity of GED compared with ICA, the single IC identified by Töllner et al. (2017) may itself be a conglomeration of multiple functionally similar, though linearly dissociable, conflict-related theta sources.

The functional and theoretical implications of our findings warrant further consideration. Components were characterized by linear independence, low synchrony, low temporal correlations, and insensitivity of causal coupling to task features, all providing evidence against computational cooperation. Ergo, the generators are likely to perform independent computations. We can thus rule out that these sources reflect response options which compete with one another through mutual inhibition, one of the prevailing theoretical accounts of response conflict processing (Botvinick et al., 2001; Yeung et al., 2004).

What, then, may be the use of these parallel independent computations? In one possible scenario, the computations may be functionally equivalent to one another, suggesting that the 
theta generators harness comparable microcircuitry. It has been proposed that response conflict detection and signaling are achieved by specialized theta-resonant microcircuitry within MFC (Cohen, 2014a). Indeed, the presence of multiple sources of conflict theta could reflect the parallel recruitment of assemblies throughout a general neural substrate that is suited to conflict detection. Such parallelism might serve purposes along the lines of consensus or redundancy processing, or the concurrent processing of differing afferent inputs.

In another scenario, the different generators may implement different computations. It is possible that response conflict appears as a single phenomenon at the cognitive/behavioral level but is implemented by a functionally diverse range of circuits operating in the theta range. Indeed, response conflict is associated with activation in a range of subregions along the medial wall of the PFC (Ridderinkhof et al., 2004).

A third explanation may be that response conflict is implemented through the parallel recruitment of brain networks. In this scenario, each component is driven not by a single anatomic source, but by a network of sources distributed throughout cortical circuits (and possibly subcortical circuits) (see, e.g., Boran et al., 2019). This explanation would account for the more lateral MEG findings (Figs. 1D, 3D). Distinct extra-MFC networks, making distinct contributions to the task (potentially related to working memory, attention, motor control, etc.), could appear as distinct components.

From the previous two points, it follows that the midfrontal theta dimensions identified here may be related to different aspects of the conflict task. This possibility is consistent with differences in neural dynamics being observed for stimulus versus response conflict (Nigbur et al., 2012), the modality in which the stimuli are presented (Donohue et al., 2012; Castro et al., 2018), the complexity of the response mapping (Donohue et al., 2016), and the speed-accuracy trade-off (Pastötter et al., 2012). Furthermore, the exact localization of conflict theta often depends on the task and on stimulus modality (Ridderinkhof et al., 2004). It is possible that our dimensions originate from spatially distinct midfrontal structures that cannot be distinguished with the spatial resolution of MEG/EEG, but that can be accessed through linear decomposition. By systematically varying or isolating task features, future studies may be able to determine which dimensions of midfrontal theta, if any, covary with different task or conflict requirements.

In interpreting and generalizing the results of this study, some limitations should be kept in mind. First, statistically separable sources do not equate to anatomically local generators of activity. A statistical source is at its core a spatial filter, the identification of which may have been driven by a single anatomic generator or by distributed anatomic generators that were strongly coupled. As such, the identified components clearly reflected a combination of electrodes and sensors with strong correlational patterns, but are not necessarily driven by a single spatially restricted dipole. Nonetheless, the decomposition of midfrontal theta into multiple components demonstrates that conflictrelated midfrontal theta is distributed in a multidimensional subspace, as opposed to being captured by a single statistical source (see also de Cheveigné and Parra, 2014).

Furthermore, our analyses necessitated some design choices. We selected the theta frequency band $(5-7 \mathrm{~Hz})$ as our frequency range of interest, based on strong a priori expectations about the manifestation of response conflict. This selection means that our analysis could not capture potentially conflict-relevant sources in other frequencies. Likewise, our explicit template-based selection of midfrontal sources may have excluded relevant sources with different topographical manifestations, such as those that are driven by parietal, visual, or motor sources. However, the selection was applied only to the EEG topographies, while the MEG topographies were left unconstrained. It is thus possible that we captured spatially distributed networks of which the non-midfrontal dipoles were obscured to EEG.

In this work, we have challenged the notion that midfrontal conflict theta is a singular phenomenon. Advanced source separation methods on a multimodal dataset have revealed the presence of multiple theta sources in each subject. Thus, conflict theta, which appears as a single process without close inspection, consists of the composite activity of multiple dissociable theta generators that are likely to implement independent computations. This conceptualization of response conflict theta as a multidimensional phenomenon allows for new functional accounts of conflict processing. Future studies should validate the existence and investigate the nature of these theta sources in more detail, for example, in electrophysiological data recorded at smaller spatial scales.

\section{References}

Barnett L, Seth AK (2014) The MVGC multivariate Granger causality toolbox: a new approach to Granger-causal inference. J Neurosci Methods 223:50-68.

Benjamini Y, Hochberg Y (1995) Controlling the false discovery rate: a practical and powerful approach to multiple testing. J R Stat Soc B 57:289300.

Boran E, Fedele T, Klaver P, Hilfiker P, Stieglitz L, Grunwald T, Sarnthein J (2019) Persistent hippocampal neural firing and hippocampal-cortical coupling predict verbal working memory load. Sci Adv 5:eaav3687.

Botvinick MM, Braver TS, Barch DM, Carter CS, Cohen JD (2001) Conflict monitoring and cognitive control. Psychol Rev 108:624-652.

Buzsáki G, Draguhn A (2004) Neuronal oscillations in cortical networks. Science 304:1926-1929.

Castro L, Soto-Faraco S, Morís Fernández L, Ruzzoli M (2018) The breakdown of the Simon effect in cross-modal contexts: EEG evidence. Eur J Neurosci 47:832-844.

Cavanagh JF, Frank MJ (2014) Frontal theta as a mechanism for cognitive control. Trends Cogn Sci 18:414-421.

Cavanagh JF, Zambrano-Vazquez L, Allen JJB (2012) Theta lingua franca: a common mid-frontal substrate for action monitoring processes. Psychophysiology 49:220-238.

Cohen MX (2014a) A neural microcircuit for cognitive conflict detection and signaling. Trends Neurosci 37:480-490.

Cohen MX (2014b) Analyzing neural time series data: theory and practice. Cambridge, MA: Massachusetts Institute of Technology.

Cohen MX (2017a) Comparison of linear spatial filters for identifying oscillatory activity in multichannel data. J Neurosci Methods 278:1-12.

Cohen MX (2017b) Multivariate cross-frequency coupling via generalized eigendecomposition. eLife 6:e21792.

Cohen MX, Cavanagh JF (2011) Single-trial regression elucidates the role of prefrontal theta oscillations in response conflict. Front Psychol 2:30.

Cohen MX, Donner TH (2013) Midfrontal conflict-related theta-band power reflects neural oscillations that predict behavior. J Neurophysiol 110: 2752-2763.

Cohen MX, Gulbinaite R (2017) Rhythmic entrainment source separation: optimizing analyses of neural responses to rhythmic sensory stimulation. Neuroimage 147:43-56.

Cohen MX, Ridderinkhof KR (2013) EEG source reconstruction reveals frontal-parietal dynamics of spatial conflict processing. PLoS One 8:e57293.

Cohen MX, van Gaal S (2014) Subthreshold muscle twitches dissociate oscillatory neural signatures of conflicts from errors. Neuroimage 86:503-513.

de Cheveigné A, Arzounian D (2015) Scanning for oscillations. J Neural Eng 12:066020.

de Cheveigné A, Parra LC (2014) Joint decorrelation, a versatile tool for multichannel data analysis. Neuroimage 98:487-505. 
Delorme A, Makeig S (2004) EEGLAB: an open source toolbox for analysis of single-trial EEG dynamics including independent component analysis. J Neurosci Methods 134:9-21.

Donohue SE, Liotti M, Perez R, Woldorff MG (2012) Is conflict monitoring supramodal? Spatiotemporal dynamics of cognitive control processes in an auditory Stroop task. Cogn Affect Behav Neurosci 12:1-15.

Donohue SE, Appelbaum LG, McKay CC, Woldorff MG (2016) The neural dynamics of stimulus and response conflict processing as a function of response complexity and task demands. Neuropsychologia 84:14-28.

Gratton G, Coles MG, Donchin E (1992) Optimizing the use of information: strategic control of activation of responses. JExp Psychol Gen 121:480-506.

Groppe DM, Makeig S, Kutas M (2009) Identifying reliable independent components via split-half comparisons. Neuroimage 45:1199-1211.

Hanslmayr S, Pastötter B, Bäuml KH, Gruber S, Wimber M, Klimesch W (2008) The electrophysiological dynamics of interference during the Stroop task. J Cogn Neurosci 20:215-225.

Haufe S, Meinecke F, Görgen K, Dähne S, Haynes JD, Blankertz B, Bießmann F (2014) On the interpretation of weight vectors of linear models in multivariate neuroimaging. Neuroimage 87:96-110.

Hayton JC, Allen DG, Scarpello V (2004) Factor retention decisions in exploratory factor analysis: a tutorial on parallel analysis. Organ Res Methods 7:191-205.

Kahana MJ, Seelig D, Madsen JR (2001) Theta returns. Curr Opin Neurobiol 11:739-744.

Leuthold H (2011) The Simon effect in cognitive electrophysiology: A short review. Acta Psychol 136:203-211.

Nigbur R, Ivanova G, Stürmer B (2011) Theta power as a marker for cognitive interference. Clin Neurophysiol 122:2185-2194.

Nigbur R, Cohen MX, Ridderinkhof KR, Stürmer B (2012) Theta dynamics reveal domain-specific control over stimulus and response conflict. J Cogn Neurosci 24:1264-1274.
Nikulin VV, Nolte G, Curio G (2011) A novel method for reliable and fast extraction of neuronal EEG/MEG oscillations on the basis of spatio-spectral decomposition. Neuroimage 55:1528-1535.

Nunez PL, Srinivasan R (2006) Electric fields of the brain. Oxford: Oxford UP.

Pastötter B, Berchtold F, Bäuml KH (2012) Oscillatory correlates of controlled speed-accuracy tradeoff in a response-conflict task. Hum Brain Mapp 33:1834-1849.

Pastötter B, Dreisbach G, Bäuml KH (2013) Dynamic adjustments of cognitive control: oscillatory correlates of the conflict adaptation effect. J Cogn Neurosci 25:2167-2178.

Ridderinkhof KR, Ullsperger M, Crone EA, Nieuwenhuis S (2004) The role of the medial frontal cortex in cognitive control. Science 306:443-447.

Töllner T, Wang Y, Makeig S, Müller HJ, Jung TP, Gramann K (2017) Two independent frontal midline theta oscillations during conflict detection and adaptation in a Simon-type manual reaching task. J Neurosci 37:2504-2515.

van de Vijver I, Ridderinkhof KR, Cohen MX (2011) Frontal oscillatory dynamics predict feedback learning and action adjustment. J Cogn Neurosci 23:4106-4121.

Verguts T (2017) Binding by random bursts: a computational model of cognitive control. J Cogn Neurosci 29:1103-1118.

Wang XJ (2010) Neurophysiological and computational principles of cortical rhythms in cognition. Physiol Rev 90:1195-1268.

Womelsdorf T, Valiante TA, Sahin NT, Miller KJ, Tiesinga P (2014) Dynamic circuit motifs underlying rhythmic gain control, gating and integration. Nat Neurosci 17:1031-1039.

Yeung N, Botvinick MM, Cohen JD (2004) The neural basis of error detection: conflict monitoring and the error-related negativity. Psychol Rev 111:931-959. 